\title{
A Practical Diversity Combining Scheme based on the Selection of Maximal Ratio Combined Signals
}

\author{
Flávio du Pin Calmon and Michel Daoud Yacoub
}

\begin{abstract}
Resumo-Este artigo apresenta um esquema geral de combinação de diversidade onde sinais combinados utilizando MRC (maximal-ratio combining) são escolhidos através de um algoritmo de combinação por seleção. Este método possui uma implementação simples e uma formulação analítica tratável. A distribuição de probabilidade, taxa de cruzamento de nível e duração média de desvanecimento na saída do combinador são analisadas, exemplificando para um ambiente Nakagami-m. O resultado principal deste trabalho, no entanto, é a derivação de uma expressão exata para a SNR na saída do combinador, considerando desvanecimento Nakagami-m. As formulações apresentadas podem ser utilizadas para dimensionamento de redes celulares.
\end{abstract}

Palavras-Chave-Combinação de Diversidade, Combinação MRC, Combinação por Seleção, Desvanecimento, Nakagami- $m$.

Abstract-This paper presents a general diversity combining scheme, in which maximal-ratio combined signals are chosen on a traditional selection combining algorithm. This method has a simple implementation and a tractable analytical formulation. A general analysis of the probability distribution, level crossing rate, and average fade duration at the output of the combiner is provided, exemplifying for a Nakagami- $m$ fading environment. The main result of the present work, however, is the derivation of an exact expression for the SNR at the output of the combiner for a Nakagami- $m$ fading scenario. The formulations derived here find a direct applicability in the dimensioning of cellular networks.

Keywords-Diversity Combining, Maximal Ratio, Pure Selection, Fading, Nakagami- $m$.

\section{INTRODUCTION}

Diversity combining techniques are largely employed in communication systems as means of improving the system performance in fading environments. Three combining methods, namely maximal-ratio combining (MRC), equal gain combining (EGC), and selection combining (SC), are the most widely investigated schemes. More recently, however, a composite technique, introduced as a "generalized combining scheme" [1], has been proposed. In this scheme, a group of signals is selected out of the total available, which are then maximalratio combined. Such a technique as well as variations of it were then explored in several other opportunities [2]-[5]. As opposed to the individual techniques (MRC and SC), the composite one, which maximal-ratio combines a group of selected signals, has an intricate mathematical treatment, dealing with order statistics [6].

The authors are with the Wireless Technology Laboratory (WissTek), Department of Communications, School of Electrical and Computer Engineering, State University of Campinas (UNICAMP), P.O. Box 6101, 13083-852, Campinas, SP, Brazil (E-mail: [fcalmon,michel]@ wisstek.org). This work was partly supported by FAPESP (07/02117-1).
In this paper, we investigate another generalized diversity scheme that is dual to the one discussed above. In such a technique, here named MRCS (MRC Selection), the outputs of $N M_{i}$-branch maximal ratio combiners, $i=1, \ldots, N$, are combined into a $N$-branch selection combiner. The MRCS scheme has two very attractive key features: (i) it is readily applied to situations where site diversity exists, such as in [7]; and (ii) it is mathematically easily tractable. In soft-handoff situations, the MRCS technique is already employed, with the serving base stations providing the MRC of the signals received from a mobile terminal, and the serving switching center carrying out the SC of the MRC signals [8]. To the best of the authors' knowledge, the analysis of such a scheme, including several expressions for a general fading scenario and simple closed-form formulations for cases of interest, has never been shown in the literature. The main result of the present work, however, is the derivation of an exact, easy-toevaluate closed-form expression for the signal-to-noise ratio (SNR) at the output of the MRCS combiner for a Nakagami$m$ fading scenario, of which a particular case is found in the literature. The formulations presented here find a direct applicability in the dimensioning of cellular networks.

The remainder of this paper is organized as follows. Section II presents general and simple closed-form expressions for: (i) the probability distribution (PDF) as well as density functions (pdf) of the signal-to-noise ratio (SNR) at the output of the MRCS combiner; (ii) level crossing rate (LCR) and average fade duration (AFD) at the output of the MRCS combiner. Such expressions are applicable to any fading scenario, but here they are specialized to the Nakagami- $m$ environment, as an example. Section III derives a closed-form expression of the average SNR at the output of the MRCS combiner for the Nakagami- $m$ environment, assuming $m_{i} M_{i}$ an integer for each selected $M_{i}$-branch MRC receiver. This expression is very general and new, having a simple computational implementation, and constitutes the main result of the present work. Section IV shows some plots of the formulations derived. Finally, Section V presents some concluding remarks and a summary of our results.

\section{MRC SELECTION - MRCS}

The MRCS is a hybrid combining scheme in which $N$ $M_{i}$-branch MRC receivers constitute inputs of an $N$-branch selection combiner. The formulations derived here assume unbalanced, independent branches operating in an arbitrary fading scenario. The formulations are then particularized for some special case. 


\section{A. Probability Distribution Function - Reliability}

Let $F_{m r c_{i}}(\gamma)$ be the probability distribution of the output SNR $\gamma$ of the $i$ th MRC combiner. Assuming the selection branches to be independent, the output SNR distribution $F_{\text {sel }}$ at the selection combiner is

$$
F_{s e l}(\gamma)=\prod_{i=1}^{N} F_{m r c_{i}}(\gamma)
$$

Of course, in (1) the branches of the MRC receiver may not be independent, and this must be accounted for in $F_{m r c_{i}}(\gamma)$. The probability density function for the SC output can be obtained by differentiating (1), which results in

$$
f_{\text {sel }}(\gamma)=\sum_{i=1}^{N} f_{m r c_{i}}(\gamma) \prod_{\substack{j=1 \\ j \neq i}}^{N} F_{m r c_{j}}(\gamma) .
$$

Note that the previous formulations assume arbitrary fading environments, for which the MRC output statistics are known. As an illustration example, consider the Nakagami- $m$ fading case, with each MRC receiver $i$ having $M_{i}$ i.i.d. branches in an scenario for which the parameters are $m_{i}$ and $\beta_{i}=\gamma_{i} / \bar{\gamma}_{i}$. Then

$$
F_{m r c_{i}}\left(\beta_{i}\right)=\frac{\Gamma\left(m_{i} M_{i}, m_{i} \beta_{i}\right)}{\Gamma\left(m_{i} M_{i}\right)}
$$

and

$$
f_{m r c_{i}}\left(\beta_{i}\right)=\frac{m_{i}^{m_{i} M_{i}} \beta_{i}^{m_{i} M_{i}-1}}{\Gamma\left(m_{i} M_{i}\right) \exp \left(m_{i} \beta_{i}\right)} .
$$

where $\Gamma(a, b)=\int_{0}^{b} x^{a-1} \exp (-x) d x$ is the incomplete Gamma function. The substitution of these expressions into (1) and (2) yields the required statistics. In particular, in a full i.i.d. scenario, then

$$
F_{\text {sel }}(\beta)=\left(\frac{\Gamma(m M, m \beta)}{\Gamma(m M)}\right)^{N}
$$

and

$$
f_{\text {sel }}(\beta)=\frac{N m^{m M} \beta^{m M-1}}{\Gamma(m M) \exp (m \beta)}\left(\frac{\Gamma(m M, m \beta)}{\Gamma(m M)}\right)^{N-1}
$$

The reliability is obtained as the complement of the PDF.

\section{B. $L C R$ and $A F D$}

The MRCS output envelope $R$ can be written as $R=$ $\max _{i=1, \ldots, N}\left\{R_{i}\right\}$, where $R_{i}$ denotes the envelope at the output of the $i$ th maximal-ratio combiner. The LCR is defined as the average number of signal crossings at a given level, in the negative or positive direction. Considering independence of the signals received by the selection combiner, the level crossing rate $N_{R}(r)$ of the MRCS output at level $r$ is given by [9]

$$
N_{R}(r)=\sum_{i=1}^{N} N_{R_{i}}(r) \prod_{\substack{j=1 \\ j \neq i}}^{N} F_{R_{j}}(r) .
$$

The expression (7) can be directly applied to fading environments with known LCR and envelope PDF at the output of each MRC receiver, denoted by $N_{R_{i}}(\cdot)$ and $F_{R_{i}}(\cdot)$, respectively.
The AFD is defined as the mean time the received envelope remains below a given threshold $r$ after crossing it in the negative direction. Considering independent branches, the AFD $T_{R}(r)$ at the output of the MRCS combiner is [10]

$$
T_{R}^{-1}(r)=\sum_{i=1}^{N} T_{R_{i}}^{-1}(r) .
$$

From the previous expression, we note that, analogously to the LCR, given the AFD $T_{R_{i}}(\cdot)$ of each MRC output, the average fade duration of the MRCS output signal can be obtained.

To illustrate expressions (7) and (8), we shall consider once again the Nakagami- $m$ fading statistics. The LCR of the MRC output for the $i$ th receiver is given by [11]

$$
N_{R_{i}}(r)=\frac{\sqrt{2 \pi} f_{m}\left(m_{i} \rho_{i}^{2}\right)^{m_{i} M_{i}-0.5}}{\Gamma\left(m_{i} M_{i}\right) \exp \left(m_{i} \rho_{i}^{2}\right)},
$$

where $f_{m}$ is the maximum Doppler shift, $\rho_{i}=r / \sqrt{\Omega_{i}}$ and $\Omega_{i}=E\left[R_{i}^{2}\right]$. The PDF for the $i$ th MRC output is

$$
F_{i}(r)=\frac{\Gamma\left(m_{i} M_{i}, m_{i} \rho_{i}^{2}\right)}{\Gamma\left(m_{i} M_{i}\right)} .
$$

Substituting (9) and (10) in (7), an exact solution for the MRCS output LCR is readily obtained. When all the selected sites have the same Nakagami parameter $m$, second-moment $\Omega_{i}$ and number of branches $M$, the level crossing rate reduces to

$N_{R}(r)=\frac{N \sqrt{2 \pi} f_{m}\left(m \rho^{2}\right)^{m M-0.5}}{\Gamma(m M) \exp \left(m \rho^{2}\right)}\left(\frac{\Gamma\left(m M, m \rho^{2}\right)}{\Gamma(m M)}\right)^{N-1}$.

The AFD for the $i$ th MRC output in a Nakagami- $m$ environment is [11]

$$
T_{R_{i}}(r)=\frac{\Gamma\left(m_{i} M_{i}, m_{i} \rho_{i}^{2}\right) \exp \left(m_{i} \rho_{i}^{2}\right)}{f_{m} \sqrt{2 \pi}\left(m_{i} \rho_{i}^{2}\right)^{m_{i} M_{i}-0.5}} .
$$

Substituting the previous equation in (8), the AFD of the signal at the MRCS output can be found in an exact manner. Considering again the scenario where all MRC receivers have equal $m, M$ and $\rho$, the AFD is given by

$$
T_{R}(r)=\frac{\exp \left(m \rho^{2}\right) \Gamma\left(m M, m \rho^{2}\right)}{N \sqrt{2 \pi} f_{m}\left(m \rho^{2}\right)^{m M-0.5}} .
$$

\section{OUTPUT MEAN SNR FOR A NAKAGAMI- $m$ ENVIRONMENT}

In this section, a closed-form expression for the average signal-to-noise ratio at the MRCS output in a Nakagami- $m$ scenario is derived, assuming the $M_{i}$ input signals at the MRC receiver $i$ to be i.i.d with mean input SNR equal to $\gamma_{0 i}$. The fading statistics is such that $\mathbf{M}_{i} \triangleq M_{i} m_{i}$ is an integer. To facilitate the notation, we define $\alpha_{i}=m_{i} / \gamma_{0 i}$. The PDF for the maximal-ratio combined signal at the $i$ th receiver is given by:

$$
F_{m r c_{i}}(\gamma)=1-\exp \left(-\alpha_{i} \gamma\right) \sum_{k=1}^{\mathbf{M}_{i}} \frac{\left(\alpha_{i} \gamma\right)^{k-1}}{(k-1) !}
$$


The mean SNR at the MRCS output can be calculated as

$$
\bar{\gamma}=\int_{0}^{\infty}\left[1-F_{\text {sel }}(\gamma)\right] d \gamma
$$

where $F_{\text {sel }}(\gamma)$ is given in (1). Define

$$
g_{j}(\gamma) \triangleq-\exp \left(-\alpha_{j} \gamma\right) \sum_{i=1}^{\mathbf{M}_{j}} \frac{\left(\alpha_{j} \gamma\right)^{i-1}}{(i-1) !}
$$

Then, from (1),

$$
F_{\text {sel }}(\gamma)=\prod_{i=1}^{N}\left[1+g_{i}(\gamma)\right]
$$

and

$$
\begin{aligned}
\bar{\gamma} & =\int_{0}^{\infty}\left[1-\prod_{i=1}^{N}\left(1+g_{i}(\gamma)\right)\right] d \gamma \\
& =-\int_{0}^{\infty} \sum_{i=1}^{2^{N}-1}\left(\prod_{j=1}^{N}\left[g_{j}(\gamma)\right]^{b_{j}(i)}\right) d \gamma
\end{aligned}
$$

where $b_{j}(i)$ is the $j$ th bit of the N-bit binary representation of $i$. Expanding the product term in (17), then

$$
\begin{aligned}
& \prod_{j=1}^{N}\left[g_{j}(\gamma)\right]^{b_{j}(i)}=\prod_{j=1}^{N}\left(-\exp \left(-\alpha_{j} \gamma\right) \sum_{k=1}^{\mathbf{M}_{j}} \frac{\left(\alpha_{j} \gamma\right)^{k-1}}{(k-1) !}\right)^{b_{j}(i)} \\
& =\exp \left(-\gamma \sum_{l=1}^{N} \alpha_{l} b_{l}(i)\right) \times \prod_{j=1}^{N}\left(\sum_{k=1}^{\mathbf{M}_{j}}-\frac{\left(\alpha_{j} \gamma\right)^{k-1}}{(k-1) !}\right)^{b_{j}(i)} .
\end{aligned}
$$

(18) can be written as

$$
\begin{aligned}
& \prod_{j=1}^{N}\left(\sum_{i=1}^{\mathbf{M}_{j}}-\frac{\left(\alpha_{j} \gamma\right)^{i-1}}{(i-1) !}\right)^{b_{j}(i)}= \\
& =\sum_{k_{1}=0}^{\left(\mathbf{M}_{1}-1\right) b_{1}(i)} \sum_{k_{2}=0}^{\left(\mathbf{M}_{2}-1\right) b_{2}(i)} \cdots \sum_{k_{N}=0}^{\left(\mathbf{M}_{N}-1\right) b_{N}(i)}\left(-\frac{\left(\alpha_{1} \gamma\right)^{k_{1}}}{k_{1} !}\right)^{b_{1}(i)} \times \\
& \quad \times\left(-\frac{\left(\alpha_{2} \gamma\right)^{k_{2}}}{k_{2} !}\right)^{b_{2}(i)} \cdots\left(-\frac{\left(\alpha_{N} \gamma\right)^{k_{n}}}{k_{N} !}\right)^{b_{N}(i)} \cdots \sum_{k_{N}=0}^{\left(\mathbf{M}_{1}-1\right) b_{1}(i)} \sum^{\left.\mathbf{M}_{N}-1\right) b_{N}(i)} \sum_{l=1}^{N} k_{l} \prod_{j=1}^{N}\left(-\frac{\alpha_{j}^{k_{j}}}{k_{j} !}\right)^{b_{j}(i)} .
\end{aligned}
$$

Substituting (19) in (17) we find that

$$
\begin{aligned}
\bar{\gamma}= & -\sum_{i=1}^{2^{N}-1} \sum_{k_{1}=0}^{\left(\mathbf{M}_{1}-1\right) b_{1}(i)} \ldots \sum_{k_{N}=0}^{\left(\mathbf{M}_{N}-1\right) b_{N}(i)} \int_{0}^{\infty} \gamma^{\sum_{l=1}^{N} k_{l} \times} \\
& \times \exp \left(-\gamma \sum_{l=1}^{N} \alpha_{l} b_{l}(i)\right) \prod_{j=1}^{N}\left(-\frac{\alpha_{j}^{k_{j}}}{k_{j} !}\right)^{b_{j}(i)} d \gamma .
\end{aligned}
$$

The result of the previous integration is

$$
\begin{array}{r}
\bar{\gamma}=-\sum_{i=1}^{2^{N}-1} \sum_{k_{1}=0}^{\left(\mathbf{M}_{1}-1\right) b_{1}(i)} \ldots \sum_{k_{N}=0}^{\left(\mathbf{M}_{N}-1\right) b_{N}(i)}(-1)^{w(i)}(\mathbb{K}) ! \times \\
\times\left(\sum_{l=1}^{N} \alpha_{l} b_{l}(i)\right)^{-1-\mathbb{K}} \prod_{j=1}^{N}\left(\frac{\alpha_{j}^{k_{j}}}{k_{j} !}\right)
\end{array}
$$

where $w(i)$ is the Hamming weight of $i$ and $\mathbb{K}=\sum_{l=1}^{N} k_{l}$.

Equation (21) presents a closed-form expression for the mean SNR at the output of a MRCS combiner in a Nakagami$m$ environment with integer $m_{i} M_{i}$. However, (21) does not have a simple algorithmic implementation, due to the fact that the number of summation terms varies with the number of $\mathrm{MRC}$ receivers (whose outputs constitute the inputs to the SC). Hence, in order to solve this, we rewrite such an expression for $\bar{\gamma}$ as

$$
\begin{array}{r}
\bar{\gamma}=-\sum_{i=1}^{2^{N}-1} \sum_{\mathbf{K} \in A(i)}(-1)^{w(i)}\left(\mathbf{1} \cdot \mathbf{K}^{\prime}\right) !\left(\boldsymbol{\alpha} \cdot \mathbf{B}_{i}^{\prime}\right)^{\left(-1-\mathbf{1} \cdot \mathbf{K}^{\prime}\right)} \times \\
\times \prod_{j=1}^{N}\left(\frac{\alpha_{j}^{k_{j}}}{k_{j} !}\right)
\end{array}
$$

where $\mathbf{B}_{i}, \mathbf{K}$ and $\boldsymbol{\alpha}$ are $N$-dimensional row vectors, whose elements are denoted by $b_{i j}, k_{j}$ and $\alpha_{j}$, respectively. $\mathbf{B}_{i}$ is composed by the bits resulting of the N-bit binary representation of $i$ and $\boldsymbol{\alpha}$ is formed by all of the $N \alpha_{i}$ 's, as defined previously. At each iteration of $i$, the vector $\mathbf{K}$ spans the set $A(i)$, defined as

$A(i)=\left\{\mathbf{K} \mid b_{i j}=0 \rightarrow k_{j}=0,0 \leq k_{j} \leq \mathbf{M}_{j}-1,1 \leq j \leq N\right\}$

For the special case $\mathbf{M}_{i}=\mathbf{M}=1$ and $\alpha_{i}=\alpha$ for every receiver, (21) reduces to

$$
\alpha \bar{\gamma}=-\sum_{i=1}^{2^{N}-1} \frac{(-1)^{w(i)}}{w(i)}=\sum_{i=1}^{N}\left(\begin{array}{c}
N \\
i
\end{array}\right) \frac{(-1)^{i+1}}{i} .
$$

Using [12, Equation 0.155.4], then

$$
\alpha \bar{\gamma}=\sum_{i=1}^{N} \frac{1}{i}
$$

Furthermore, when $m_{i}=1$ for every receiver, (25) reduces to the well-known average SNR for the selection combiner in a Rayleigh environment. Analogously, for $N=1$ and $m=1$, it is simple to verify that (21) reduces to the average SNR for the maximal-ratio combiner for Rayleigh fading case.

\section{Numerical Results AND Discussions}

In a full Nakagami-m i.i.d. scenario, (11) and (13) can be used directly to illustrate the LCR and AFD performance. Some plots are then shown in Fig. 1 for different values of $m, N$, and $M$. By comparing Fig. $1 \mathrm{a}$ with $1 \mathrm{~b}$ and $1 \mathrm{c}$ with $1 \mathrm{~d}$, it is readily seen that the number of MRC branches $(M)$ have a greater impact on the LCR and AFD statistics than the number of MRC receivers $(N)$. (A more noticeable variation 


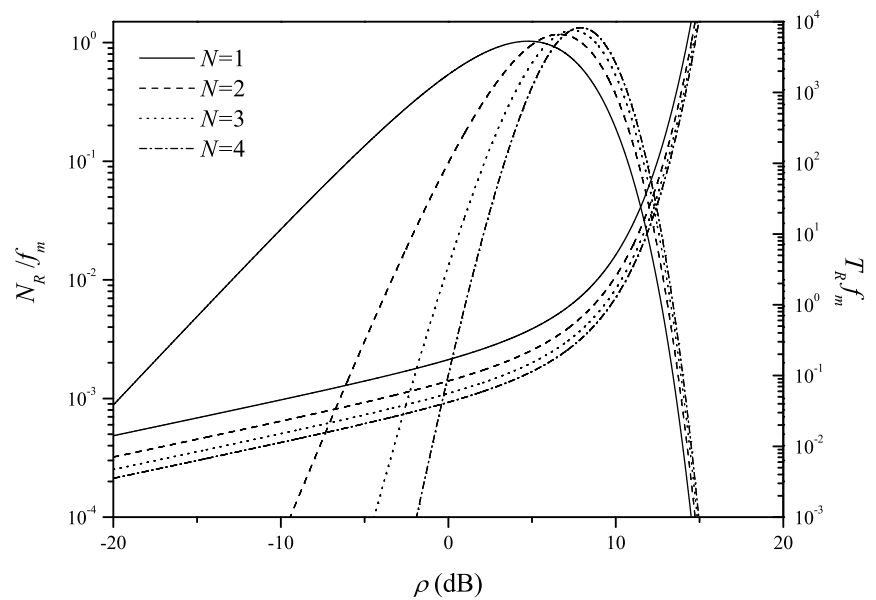

(a) $m=0.5, M=4$

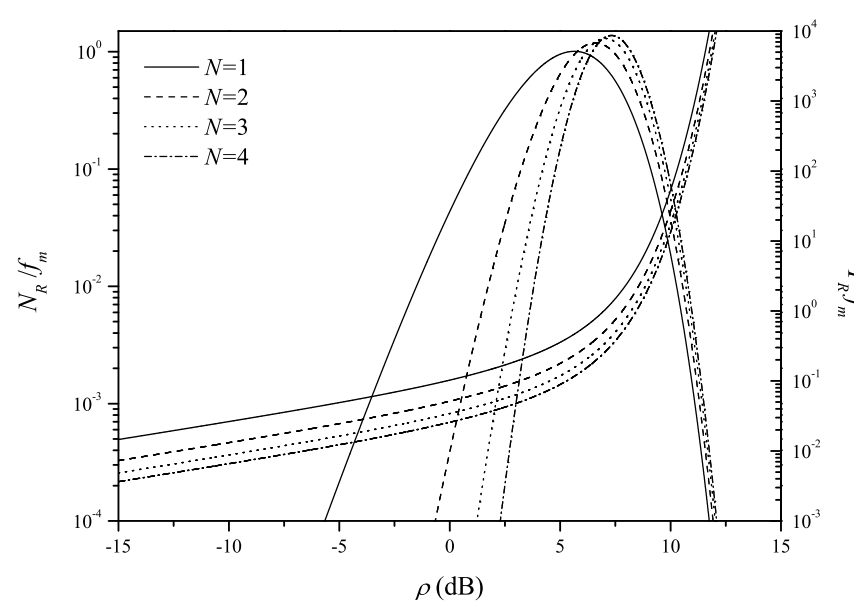

(c) $m=1.5, M=4$

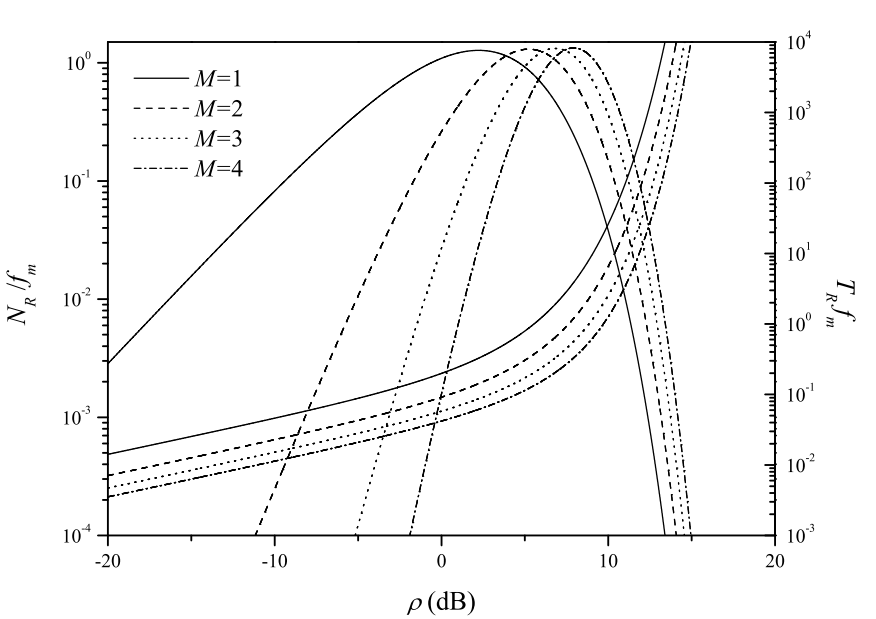

(b) $m=0.5, N=4$

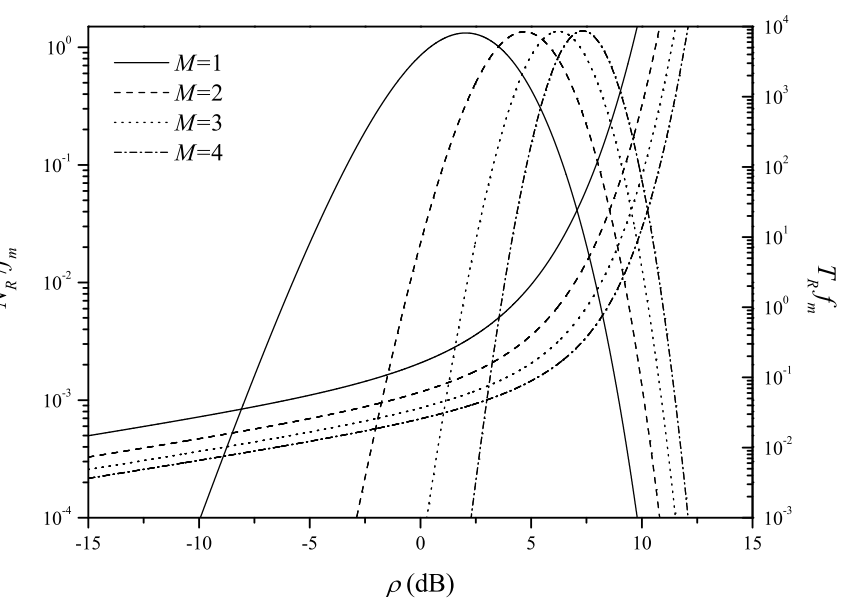

(d) $m=1.5, N=4$

Fig. 1. LCR and AFD at the output of the MRCS combiner for a Nakagami environment with parameter $m$, considering symmetric sites. In (a) and (c) the number of branches at each MRC site is maintained constant, and in (b) and (d) the number of selected sites is kept constant.

of the curves is found with the increase of $M$ rather than of N.)

Such a conclusion, however, may not apply in case an unbalanced scenario is considered, in which the mean power varies from one MRC receiver to another. In order to illustrate this fact, consider the following MRCS configurations: (i) $N=2, M=2$, and power balance; (ii) $N=2, M=3$, and power balance; (iii) $N=3, M=2$, and power balance; (iv) $N=3, M=2$, and power unbalance with two MRC receivers having identical powers and the third MRC having a power 6 $\mathrm{dB}$ higher. These configurations are investigated for different values of $m$. For the balanced case, the formulations are those used as before whereas for the more general unbalanced case (7) and (8) are the required expressions.

The resulting curves for the LCR and AFD in these scenarios are shown in Fig. 2. Observing the performance variations from configuration (i) to (ii) and comparing them with those of (iii) to (iv) we see that the latter one are much more substantial than the former one. These results show the usefulness of the formulations derived here.

Now we turn our attention to the mean SNR. Refer to Fig. 3, in which the results for $m=0.5$ and 1 are presented, considering $m_{i}=m, M_{i}=M$ and $\gamma_{0 i}=\gamma_{0}$ for each receiver. The mean output SNR normalized with respect to the branch mean SNR is depicted as a function of the number of sites $(N)$ and number of branches per site $(M)$ for each environment. As can be observed, for any given number of MRC branches $(M)$, the differential mean power gain steadily decreases with the increase of the number of MRC receivers $(N)$. In addition, such a differential gain also decreases with the increase of $M$. On the other hand, the gain obtained with the increase of the number of MRC branches is nearly constant, i.e. approximately independent of the number of $\mathrm{MRC}$ receivers.

\section{Conclusions}

In this paper, we presented a generalized diversity combining scheme based on the selection of maximal-ratio combined signals. General formulations for the probability distribution (reliability), level crossing rate, and average fade duration were obtained, which can be applied to any fading environment with known maximal-ratio statistics. More importantly, an exact, easy-to-evaluate closed-form expression for the average 


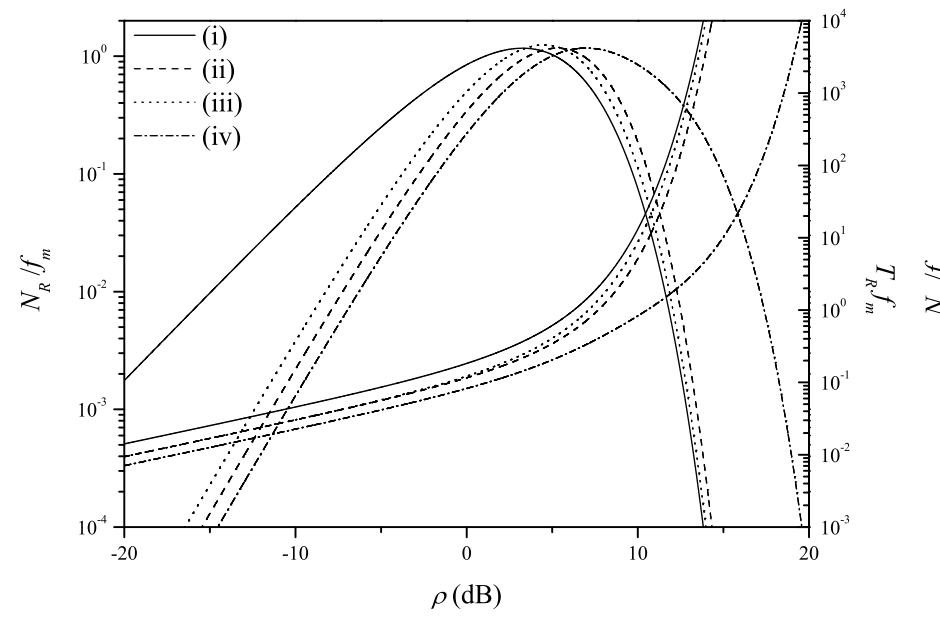

(a) $m=0.5$

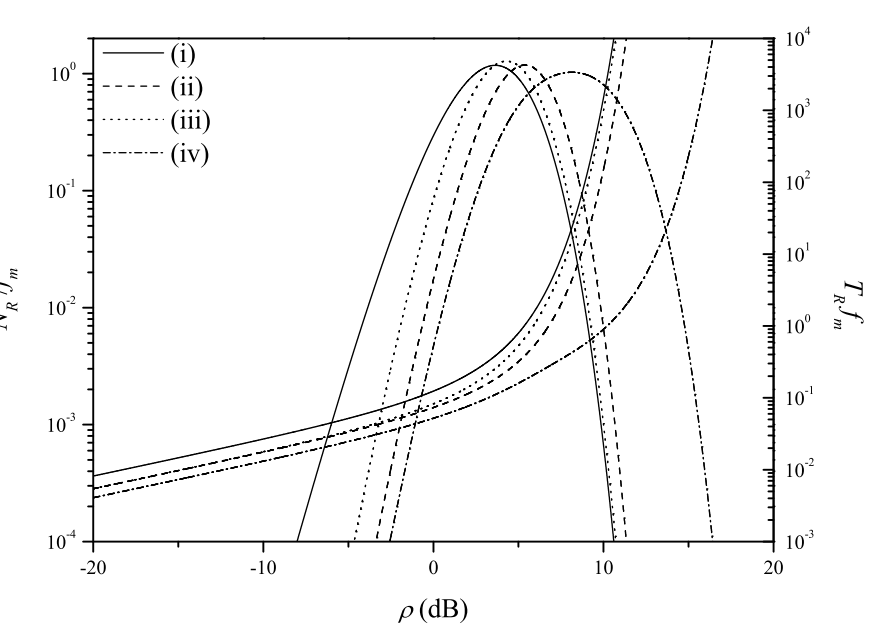

(b) $m=1.5$

Fig. 2. Comparison of the LCR and AFD at the output of the MRCS combiner considering the following scenarios: (i) $N=2, M=2$, and power balance; (ii) $N=2, M=3$, and power balance; (iii) $N=3, M=2$, and power balance; (iv) $N=3, M=2$, and power unbalance, with two MRC receivers having identical powers and the third MRC having a power $6 \mathrm{~dB}$ higher.

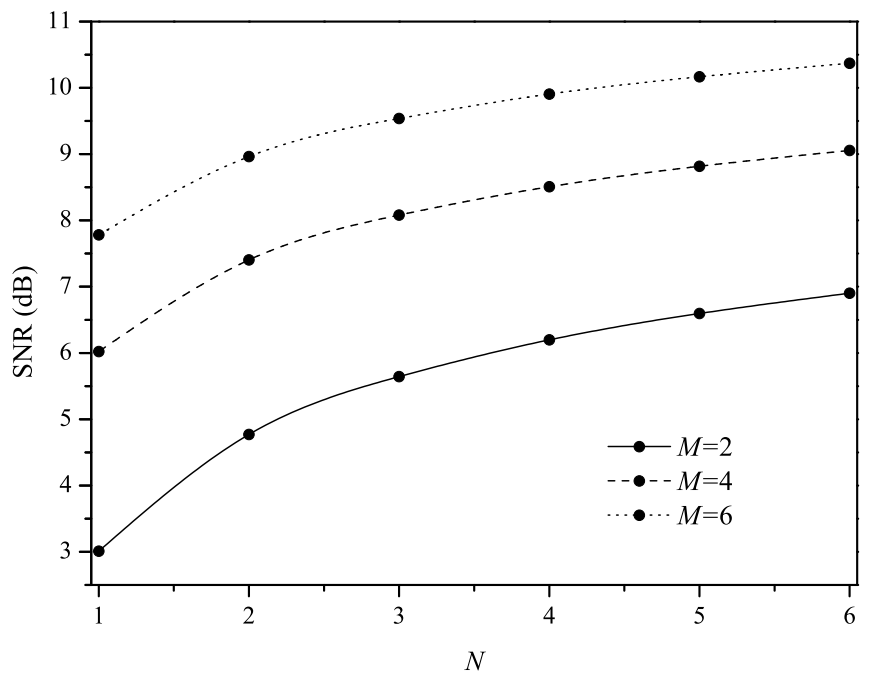

(a) $m=0.5$

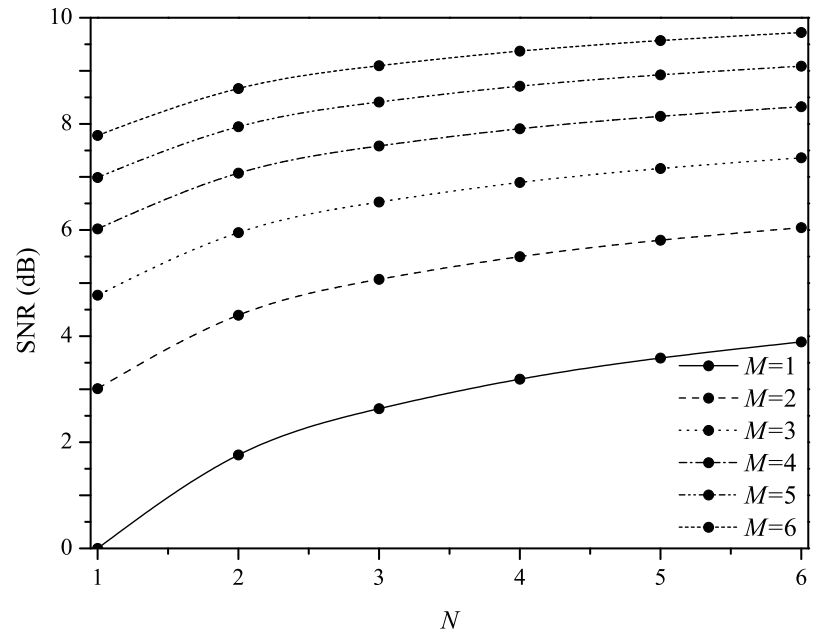

(b) $m=1$

Fig. 3. Normalized average SNR at the output of the MRCS combiner as a function of the number of selected sites, considering $M_{i}=M, m_{i}=m$ and the same mean power at each MRC receiver.

SNR at the output of the MRCS combiner in a Nakagami$m$ environment was derived, that generalizes a result found elsewhere in the literature.

\section{REFERENCES}

[1] T. Eng, N. Kong, and L. B. Milstein, "Comparison of diversity combining techniques for Rayleigh-fading channels," IEEE Trans. Commun., vol. 44, no. 9, pp. 1117-1129, Sept. 1996.

[2] N. Kong and L. B. Milstein, "Average SNR of a generalized diversity combining scheme," IEEE Commun. Lett., vol. 3, no. 3, pp. 57-59, Mar. 1999.

[3] C. R. C. M. da Silva and M. D. Yacoub, "A generalized solution for diversity combining techniques in fading channels," IEEE Trans. Microw. Theory Tech., vol. 50, no. 1, pp. 46-50, Jan. 2002.

[4] M. Win and J. Winters, "Analysis of hybrid selection/maximal-ratio combining in Rayleigh fading," IEEE Trans. Commun., vol. 47, no. 12, pp. 1773-1776, Dec. 1999.

[5] L. Yang and M.-S. Alouini, "Average level crossing rate and average outage duration of generalized selection combining," IEEE Trans. Commun., vol. 51, no. 12, pp. 1997-2000, Dec. 2003.
[6] H. A. David and H. N. Nagaraja, Order Statistics, 3rd ed. WileyInterscience, Aug. 2003.

[7] A. D. Panagopoulos, E. M. Georgiadou, and J. D. Kanellopoulos, "Selection combining site diversity performance," IEEE Commun. Lett., vol. 11 , no. 10 , pp. 787-789, Oct. 2007.

[8] H. Holma and A. Toskala, WCDMA for UMTS, 2nd Edition, 2nd ed. John Wiley \& Sons, Sept. 2002.

[9] L. Yang and M.-S. Alouini, "Level crossing rate over multiple independent random processes - an extension of the applicability of the rice formula," in Proc. IEEE Global Telecommun. Conf., vol. 3, Dec. 2003, pp. 1644-1648.

[10] D. B. da Costa, J. C. S. S. Filho, M. D. Yacoub, and G. Fraidenraich, "Crossing rates and fade durations for diversity-combining schemes over $\alpha-\mu$ fading channels," IEEE Trans. Wireless Commun., vol. 6, no. 11, Nov. 2007.

[11] M. D. Yacoub, C. R. C. M. da Silva, and J. E. V. Bautista, "Secondorder statistics for diversity-combining techniques in Nakagami-fading channels," IEEE Trans. Veh. Technol., vol. 50, no. 6, pp. 1464-1470, Nov. 2001.

[12] L. S. Gradshteyn and I. M. Ryzhik, Table of Integrals, Series and Products, 6th ed. San Diego, CA: Academic Press, 2000. 